Recepción: 12/10/2018

Aceptación: $10 / 12 / 2018$

Publicación: $20 / 01 / 2019$

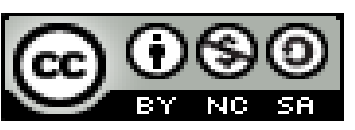

Ciencias de la educación

Artículo de Investigación

\title{
Mitos y realidades sobre la tecnología en las Universidades en el Ecuador
}

\section{Myths and realities about technology in Universities in Ecuador}

\section{Mitos e realidades sobre a tecnologia nas universidades do Equador}

Leopoldo V. Venegas-Loor ${ }^{\mathrm{I}}$ ingvenegasloor@gmail.com

Paola Y. Moreira-Aguayo ${ }^{\text {II }}$ paola.moreira@unesum.edu.ec

\section{Correspondencia: ingvenegasloor@gmail.com}

\footnotetext{
${ }^{\text {I } M a g i ́ s t e r ~ e n ~ E v a l u a c i o ́ n ~ y ~ A u d i t o r i a ~ d e ~ S i s t e m a s ~ T e c n o l o ́ g i c o s, ~ I n g e n i e r o ~ e n ~ C o m p u t a c i o ́ n ~ y ~ R e d e s, ~}$ Doctorando en Educación en la Universidad Católica Andrés Bello, Caracas, Venezuela; Docente de la Universidad Estatal del Sur de Manabí, Jipijapa, Ecuador.

II Magíster en Enseñanza del Idioma Inglés, Licenciada en Ciencias de la Educación Especialidad Inglés, Doctorando en Educación en la Universidad Católica Andrés Bello, Caracas, Venezuela; Docente de la Universidad Estatal del Sur de Manabí, Jipijapa, Ecuador.
} 


\section{Resumen}

La Universidad es uno de los ámbitos que más mitos ha amparado sobre el influjo de la tecnología. Para conocer un poco mejor la Universidad del futuro es bueno acercarse a estas creencias y ver si se han cumplido. Para conocer mejor la Universidad contemporánea es importante acercarse a los mitos que se han creado en torno a la tecnología, entendiendo que las instituciones educativas ven en el mundo digital una gran ayuda, aunque su implantación se esté llevando a un paso lento. En este ensayo, se hace un análisis documental de tipo hermenéutico acerca de los mitos y realidades sobre la tecnología en las universidades del Ecuador. Entre sus resultados, se puso percibir que, si bien la tecnología moderna tiene un gran potencial para mejorar la enseñanza y el aprendizaje, convertir ese potencial en realidad es una tarea compleja y multifacética.

Palabras clave: mitos tecnológicos; realidades tecnológicas; unidades educativas y enseñanza.

\section{Abstract}

The University is one of the areas that more myths has covered about the influence of technology. To know a little better the University of the future is good approach to these beliefs and see if they have complied. To learn more about the contemporary University it is important to approach the myths that have been created around the technology, understanding that educational institutions see in the digital world a great help even if its implementation is being a slow pace. In this essay, is a documentary hermeneutic analysis about the myths and realities about the technology in the universities of the Ecuador. Among their findings, put perceive that although modern technology has great potential to improve teaching and learning, turning that potential into reality is complex and multifaceted.

Key words: technological myths; technological realities; educational units; and teaching.

\section{Resumo}

A Universidade é uma das áreas que tem apoiado mais mitos sobre a influência da tecnologia. Para conhecer um pouco melhor a Universidade do futuro, é bom abordar essas crenças e ver se elas foram cumpridas. Para conhecer melhor a universidade contemporânea, é importante abordar os mitos que foram criados em torno da tecnologia, entendendo que as instituições educacionais 
vêem uma grande ajuda no mundo digital, embora sua implementação esteja dando passos lentos. Neste ensaio, faz-se uma análise documental hermenêutica sobre os mitos e realidades sobre a tecnologia nas universidades do Equador. Entre seus resultados, percebeu-se que, embora a tecnologia moderna tenha um grande potencial para melhorar o ensino e a aprendizagem, converter esse potencial em realidade é uma tarefa complexa e multifacetada.

Palavras-chave: mitos tecnológicos; rea; unidades de ensino e ensino.

\section{Introducción}

Estamos en medio de una explosión de tecnología digital multimedia-computadoras y todo eso va con ellos, en las unidades educativas de todo el país, propulsado por el gobierno nacional y el Ministerio de Educación (2017), e iniciativas locales, las universidades gastaron un estimado de \$ 6,9 mil millones en 2017 en computadoras, servidores, enrutadores, cableado, acceso a Internet, software y todo lo demás involucrado en hacer que la tecnología moderna esté disponible. Los fondos de educación están mejorando las líneas de fondo de Intel, Microsoft, Apple, Cisco, IBM y otras compañías de alta tecnología.

Pero, ¿Recibiremos un "retorno de la inversión" adecuado para el resultado educativo? Es decir, ¿Esta tecnología mejorará la educación de un gran número de estudiantes? ¿Será que nuestros sistemas educativos sean más efectivos y eficientes? ¿Ayudará a las universidades a mejorar y preparar a los estudiantes para la vida en el siglo 21 ?

Al comenzar este nuevo siglo, la inversión en tecnología para las universidades se asemeja a las inversiones que se realizan en muchas empresas de Internet. En ambos casos, las inversiones se basan en el potencial de las nuevas tecnologías, con la esperanza de que este potencial se cumplirá en los próximos años. $\mathrm{Y}$ en ambos casos las inversiones involucran riesgos significativos y puede estar muy lejos de generar rendimientos adecuados. Maximizar nuestra inversión en tecnología requiere una visión clara de nuestros objetivos y el desarrollo de planes para alcanzarlos.

Sin embargo, desafortunadamente, de acuerdo a lo planteado por Álvarez (2012) la rápida entrada de tecnología en las universidades está, en muchos casos, corriendo por delante de la visión educativa y una planificación cuidadosa, necesario para poner la tecnología en buen uso. De 
hecho, lo que se hace a menudo se basa en conceptos erróneos o mitos sobre lo que se requiere para obtener beneficios educativos sustanciales.

Entre estos mitos, sería que al poner computadoras en las universidades mejoraría directamente el aprendizaje; más computadoras darán como resultado un mayor aprendizaje. Cabe entender, que las computadoras son herramientas potentes y flexibles que pueden mejorar enseñando y aprendiendo de innumerables maneras. Sin embargo, el valor de una computadora, como el de cualquier herramienta, depende de los propósitos para los que sirve y qué tan bien se usa. Las computadoras pueden ser utilizadas de manera positiva, como permitir que el aprendizaje sea más atractivo, abordar las necesidades de los estudiantes individuales, proporcionar acceso a una gran cantidad de información y alentar a los estudiantes para explorar y crear, o de maneras negativas, como jugar juegos sin sentido, acceder a materiales inapropiados o aislar estudiantes. (Semenov, 2005)

Muchas computadoras en las universidades, no están siendo utilizadas de maneras que mejoren significativamente la enseñanza y el aprendizaje. Ahí hay muchas razones para esto, incluidas las siguientes:

- Los docentes no han recibido la capacitación adecuada y apoyo para integrar la tecnología en el núcleo el día de la instrucción en el aula actual, por lo que se usan computadoras alrededor del trabajo principal de la clase-para recompensar a los estudiantes que completan su trabajo rápidamente, y proporcionar simulacros a los estudiantes que están luchando con habilidades específicas, o para especiales ocasionales. Si bien estos usos son beneficiosos, no lo hacen justificar el tamaño de la inversión. (Tejedor, 2006)

- Los docentes a menudo no tienen un software que admita mayores objetivos del currículo, consistente con sus enfoques para la enseñanza, y que este bien diseñado para el uso en el aula. Cuando poseen un buen software educativo, encontrar y obtener lo que necesita para ejecutar en las computadoras que tiene, y para encajar en su plan de estudios, sigue siendo difícil en muchos casos.

- El soporte técnico a menudo es insuficiente, por lo que los problemas técnicos ocurren, y en la mayoría de los casos los docentes y los estudiantes no los pueden resolver, puede haber largas demoras antes de que el técnico esté disponible para abordarlo. Así los 
maestros interpretan que no pueden depender de la tecnología, por lo que en la mayoría de los casos no planee usarlo para propósitos importantes en el aula.

- Las formas en que las computadoras están disponibles a menudo son inconsistentes con enfoques de los docentes sobre la planificación curricular y el manejo del aula. Algunas unidades educativas han estado colocando computadoras en cada aula, apuntando a una eventual relación de una computadora por cada seis estudiantes. Esto requiere que los maestros organicen actividades diarias para que algunos estudiantes puedan trabajar en las computadoras mientras otros se dedican a otras tareas: un estilo de gestión del aula que puede ser nuevo para muchos maestros, especialmente por encima del nivel primario. En las universidades sin computadoras en los salones, los maestros tienen que mover la clase a un laboratorio de computación, que debe programarse con mucha antelación. Dado que esta situación hace que sea difícil integrar las computadoras en el flujo de lecciones, a menudo anima a los profesores a tratar las actividades de la computadora como eventos especiales, en lugar de ser centrales para el plan de estudios. (Tejedor, 2006)

- Al desarrollar materiales curriculares, los editores no han podido asumir que las universidades tienen suficientes computadoras o experiencia docente para hacer uso de tecnología central para el plan de estudios. Por lo tanto, típicamente han incluido las actividades de la computadora solo como complementos opcionales para otras tareas de la clase.

La realidad es que toda esta tecnología costosa rendirá poco retorno educativo hasta que las universidades y los distritos aborden la necesidad de profesionales capacitados, soporte técnico, disponibilidad de software apropiado, aula, gestión e integración curricular, ante ello cabe preguntarse:

\section{¿Qué propósitos educativos deberían tener las computadoras en el aula?}

Cuando exploramos esta pregunta clave, a menudo encontramos muchos puntos de vista implícitos diferentes dentro de una escuela o distrito. A menos que se articulen y aclaren, y se llegue a un consenso, las opiniones divergentes pueden conducir a expectativas contradictorias, enfoques distanciados para implementar tecnología y criterios para evaluar su impacto, todo lo cual puede crear barreras para avanzar de manera efectiva. (Litwin, 1998) 
Los objetivos más comunes para usar la tecnología en las universidades incluyen los siguientes argumentos:

- Mejorar la adquisición de habilidades básicas de matemáticas, lectura y escritura por parte de los estudiantes, y conocimiento de contenido en áreas temáticas específicas, lo que lleva a puntajes más altos en las pruebas estandarizadas. Este objetivo a menudo lleva al uso de programas de práctica y sistemas integrados de aprendizaje (que brindan lecciones y cuestionarios en línea, ajustados al ritmo de las lecciones para cada estudiante) y los complementos de software para los libros de texto.

- Motivar a los estudiantes. Este objetivo a menudo se basa en la opinión de que las universidades deben usar multimedia, materiales visualmente ricos para capturar el interés de los estudiantes que crecen en un mundo intensivo en medios. Además, la tecnología puede ayudar a los docentes a proporcionar múltiples caminos para aprender a adaptarse a los estilos de aprendizaje y fortalezas de los estudiantes individualmente. Puede permitir a los estudiantes trabajar con mayor autonomía, colaborar con sus compañeros y docentes, y obtener acceso a más información relacionada con sus propios intereses.

- Ampliar los objetivos curriculares, agregando más resolución de problemas, investigación, proyectos, aprendizaje basado y trabajo colaborativo. Este objetivo a menudo lleva a los estudiantes a realizar simulaciones, buscando información en la web, preparar informes y presentaciones usando procesadores de texto, bases de datos, herramientas gráficas de computadora y softwares de presentación multimedia.

- Mejor preparación de los estudiantes para el mercado laboral. Este objetivo a menudo lleva a las universidades a agregar estudios sobre el uso de la tecnología dentro del currículo, para que los estudiantes aprendan a usar el teclado, operaciones informáticas, y aplicaciones estándar tales como procesadores de texto y bases de datos. Sin embargo, este enfoque no aborda las principales necesidades articuladas por los líderes empresariales, que están preocupados de que sus solicitantes de empleo tengan fuertes habilidades en lectoescritura, aritmética y resolución de problemas; saber cómo reunir, organizar y analizar información; comunicarse bien; trabajar con éxito en equipos colaborativos; y ser capaz de aprender de manera efectiva. 


\section{Conclusiones}

Muchos creen que nuestro mundo cambiante requiere que reconsideremos la estructura y cultura de nuestras universidades y nuestras aulas, junto con lo que enseñamos y cómo lo enseñamos. Visiones del futuro varían ampliamente, pero la mayoría presentan una mayor autonomía estudiantil, más colaborativa, conexiones más globales, aprendizaje más rico. Recursos que los libros de texto tradicionales se les dificultan desarrollar en los estudiantes.

Por supuesto, un diseño curricular que tome en cuenta estos problemas y los aborde como objetivos a cumplir permitirá que minimicen las situaciones que entorpecen el correcto uso de los medios tecnológicos, los objetivos educativos deben ser clarificados y los planes para comprar, usar y evaluar el impacto de la tecnología deben ser desarrollados para ajustarse a esos objetivos.

Casi todas las universidades cuentan con un plan tecnológico, a menudo desarrollado como requisito para ser acreditadas. Por lo general, estos planes especifican una visión de tres a cinco años de qué hardware, software y capacidad de red serán comprados, junto con algunos planes sobre capacitación docente, asistencia técnica y mantenimiento, políticas de uso aceptable y presupuesto. Algunos planes también abordan la integración tecnología en el plan de estudios, evaluando el impacto de la tecnología en la enseñanza y aprendizaje, y revisar y actualizar el plan, pero, desafortunadamente, estos elementos críticos a menudo reciben solo atención superficial.

Para usar la tecnología de manera efectiva debemos integrarla en los planes de educación especial, planes curriculares, planes de desarrollo profesional, y todos los otros planes formulados por las universidades. Los retornos educativos significativos requieren que la tecnología sea vista como herramienta para cumplir objetivos educativos centrales, no como la definición de un nuevo conjunto de objetivos separados.

El tema central que subyace a todos estos mitos es que, si bien la tecnología moderna tiene un gran potencial para mejorar la enseñanza y el aprendizaje, convertir ese potencial en realidad es una tarea compleja y multifacética. El determinante clave de nuestro éxito no será el número de computadoras compradas o cables instalados, sino cómo definimos las visiones, preparamos y apoyamos a los docentes, el diseño el plan de estudios, así como abordar cuestiones de equidad y responder al mundo rápidamente cambiante. 


\section{Referencias Bibliográficas}

Álvarez, G. (2012). Las nuevas tecnologías en el contexto universitario: sobre el uso de blogs para desarrollar las habilidades de lectoescritura de los estudiantes. . RUSC. Universities and Knowledge Society Journal, 9(2).

Educación, M. (2017). Ministerio de educación. Obtenido de Obtenido de Ecuador ama la vida: http://educacion. gob. ec/unidades-educativas-del-milenio.

Litwin, E. (1998). Tecnología educativa. . Paidc"s.

Semenov, A. (2005). Las Tecnologías de la información y la comunicación en la enseñanza: Manual para docentes o cómo crear nuevos entornos de aprendizaje abierto por medio de las TIC.

Tejedor, F. J.-R. (2006). Competencias de los profesores para el uso de las TIC en la enseñanza. Análisis de sus conocimientos y actitudes. . Revista española de pedagogía, 21-43. 